\title{
Desarrollo e implementación de un sistema de costos de calidad en una empresa del sector automotriz que permite cuantificar y detectar las oportunidades de mejora
}

\section{RESUMEN}

En este estudio se propone implementar un sistema de costos de calidad basado en la cuantificación monetaria de los costos de prevención, evaluación y fallas que permita: hacer más objetiva la toma de decisiones respecto a la prioridad de los problemas a resolver, que el personal de la empresa modifique su cultura de calidad basada en un cambio de perspectiva respecto a la consecuencia que tienen los problemas que se presentan de forma cotidiana, y tener bases de comparación más objetivas con otras unidades de la empresa y con respecto a la competencia. El detalle que se presenta en el desarrollo del sistema de calidad en un área piloto de una empresa del ramo automotriz permitirá visualizar con objetividad las bondades y dificultades de este tipo de sistemas.

Palabras clave: costos de calidad, evaluación, fallas internas, fallas externas, prevención

DEVELOPMENT AND IMPLEMENTATION OF A SYSTEM OF QUALITY COSTS IN A COMPANY OF THE AUTOMOTIVE SECTOR THAT ALLOWS YOU TO DETECT AND QUANTIFY OPPORTUNITIES FOR IMPROVEMENT

\section{ABSTRACT}

In this study, we proposed to implement a system of quality cost. The system is based on the quantification of the monetary costs of prevention, assessment and failures that allows making an objective decision with respect to the priority of the problems to solve. It also allows to change the quality culture of the company personnel based on a change in the perspective of the consequences that daily problems do, and to have bases for a more objective comparison with other units of the company and with regard to competition. Detail arising in the development of the quality system in a pilot area of a company in the automotive industry will allow to view with objectivity the benefits and difficulties of this type of systems.

Keywords: assessment, external failure, internal failure, prevention, quality costs

\section{INTRODUCCIÓN}

En el mercado globalizado de hoy en día es de vital importancia para las empresas mantener la mejora continua en sus procesos. Esto le proporciona un ventaja fundamental ya que los productos y servicios hechos bien desde el inicio implica costos más bajos y productividad más elevada, brindando al mismo tiempo satisfacción a sus clientes.

La mayoría de las empresas promueven la calidad para cumplir con las especificaciones y satisfacer al cliente. De este modo se logra tener éxito y aumentar la competitividad de la empresa. A medida que la competitividad se incrementa, disminuye la posibilidad de obtener más utilidades con aumentos de precios, así que, la reducción de los costos de producción se ha convertido en la forma más segura para incrementar las utilidades; por esto se dice que la reducción de costos es una buena fuente de ingreso, siempre y cuando ésta no disminuya la calidad del producto o servicio. Todo programa de mejora continua ayuda a reducir los costos asociados a la mala calidad, ya que se identifican y eliminan los elementos que disminuyen la calidad, pero además, es necesario hacerlo al menor costo. Un sistema de costos de calidad permite identificar, medir y reportar los costos asociados a la calidad y permitirá a la empresa:

- Traer a la luz áreas de oportunidad a través de una eficiente selección y ejecución de proyectos de mejora que surgen en base a una metodología práctica y entendible.

- Reducir costos y aumentar sus utilidades.

- Elevar la cultura de calidad de sus empleados cambiando su perspectiva en relación a lo que se necesita y cuesta obtenerla.

- Obtener un parámetro para la comparación con otras unidades de negocio de la empresa y/o corporativo.

- Mejorar su nivel frente a la competencia

El presente trabajo se realizó en una empresa del sector automotriz cuyo giro es la manufactura de partes automotrices. Dicha empresa forma parte de un corporativo que tiene plantas

\footnotetext{
* Dr., En Planeación Estratégica y Dirección de Tecnología, Profesor Investigador, Departamento Ingeniería Industrial, Universidad de Sonora, México. E-mail: jlozano@industrial.uson.mx

** Ingeniero Industrial y Sistemas, Maestría en Ingeniaría, Empresa: Flex M Gate Hermosillo. E-mail: rodolfokeith@gmail.com

*** Dr., En Planeación Estratégica y Dirección de Tecnología, Profesor Investigador, Departamento Ingeniería Industrial,. Universidad de Sonora, México. E-mail: ifonseca@industrial.uson.mx
} 
en América, Europa y Asia y se especializa en la producción de partes automotrices que provee a las principales armadoras a nivel mundial. Esta planta en Hermosillo, Sonora, México cuenta con procesos de soldadura, inyección de plástico, estampado, ensamble y reempaque en donde se producen partes para marcas de autos reconocidas, tales como Ford, Nissan y Toyota.

La industria automotriz se encuentra dentro de un mercado altamente competitivo, donde los errores cuestan mucho dinero y las repercusiones de éstos pueden afectar futuros negocios para la empresa. Por lo tanto, prevenir los errores y atacar los existentes forma parte de la estrategia permanente por parte de la dirección.

Hoy en día la dirección de la empresa ha mostrado un interés particular por el área de soldadura, debido a que la continua existencia de problemas han creado una situación que, aunque no pudiera catalogarse como crítica, si requiere de atención y análisis para resolver los problemas y continuar mejorando para reducir los costos que se generan a raíz de las ineficiencias existentes.

En este trabajo se propone y aplica un sistema piloto de costos de calidad, utilizando el modelo de prevención, evaluación y fallas (PEF) en el área mencionada, para que la dirección cuente con una herramienta administrativa que le sirva en la toma de decisiones sobre cuales problemas son prioritarios de resolver y le ayude a medir los niveles de mejoramiento alcanzados.

Planteamiento del problema. Como bien se sabe, la mala calidad representa tiempo, trabajo y esfuerzo que le cuesta a la empresa y representa una cantidad importante de dinero que se pierde por el simple hecho de no hacer las cosas bien. Por lo tanto, identificar, cuantificar y medir correctamente los costos relacionados con la prevención, evaluación y fallas, permitirá a la empresa identificar áreas de oportunidad a las que podrá enfocar proyectos de mejora que le permitan obtener un mejor desempeño financiero.

El problema en este estudio es que no existe un sistema que permita identificar y estimar los costos de los elementos de cada una de las categorías del costo de la mala calidad para las partes automotrices manufacturadas, adecuándolo al sistema contable de la empresa.

El objetivo general de este estudio es desarrollar un sistema de costos de calidad piloto utilizando el modelo PEF enfocado al área de producción de soldadura. Los objetivos específicos son: Identificar y clasificar cada uno de los elementos del costo de calidad existentes en el área de producción de soldadura; costear o estimar los elementos del costo de calidad identificados mediante una base y metodología contable que pueda utilizarse en la empresa; seleccionar bases o índices financieros relacionados al costo de calidad que permitan la toma de decisiones justificada para la mejora y comparación con otras empresas.

\section{METODOLOGÍA}

Se presenta una recopilación de los pasos sugeridos en la implantación de un Sistema de Costos de Calidad basado en las metodologías propuestas por Harrington (1990), Dale y Plunkett (1991), Campanella (1992), Juran y Gryna (1993) y Atkinson, Hamburg y Ittner (1994).

Primer paso, presentación a la dirección. Por medio de una reunión se le presentó a la dirección de la empresa el proyecto de un sistema de costos de calidad y se le habló de los aspectos generales y los beneficios que se pueden obtener a mediano y largo plazo. La empresa dio su consentimiento para realizar el estudio después de habérsele indicado los requerimientos de información necesarios para llevar a cabo la investigación, siempre y cuando, por cuestiones de confidencialidad, no se mostraran las cifras reales. Por lo tanto, el presente documento muestra cifras correspondientes a costos alterados en un múltiplo.

Segundo paso, selección del área piloto. Este estudio se realizó en el área de soldadura debido a que:

a) Esta área cuenta con más información y detalles de costos, y existen elementos de costos de fallas internas identificados y actualmente medidos para diferentes indicadores. b) Los indicadores actuales de calidad muestran a esta sección de la empresa como un área con mayor oportunidad para mejorar. c) La dirección de la empresa muestra particular interés en encontrar medios para mejorar la calidad y reducir los costos en las partes manufacturas con procesos de soldadura, ya que son las que tienen mayor margen de utilidad.

Tercer paso, formación de un equipo piloto. El equipo interdisciplinario que se formó consta de los miembros mostrados en el cuadro 1. Con el conocimiento colectivo de los participantes se identificaron los elementos del costo que sirvieron para el cálculo de los costos de calidad del área seleccionada. 
Cuadro 1. Equipo de trabajo.

\begin{tabular}{|l|l|}
\hline \multicolumn{1}{|c|}{ Departamento } & \multicolumn{1}{c|}{ Puesto } \\
\hline Calidad & Auditor de Calidad \\
Calidad & Ingeniero de Calidad \\
Calidad & Ingeniero CMM \\
Manufactura & Líder de Soldadura \\
Manufactura & Supervisor de Producción \\
Manufactura & Ingeniero de Procesos \\
Manufactura & Ingeniero de Soldadura \\
Manufactura & Técnico de Soldadura \\
Manufactura & Gerente de Manufactura \\
Mantenimiento & Supervisor de Mantenimiento \\
Materiales & Planeador de Materiales \\
Finanzas & Contador de Costos \\
\hline
\end{tabular}

Fuente: Elaboración propia.

Cuarto paso, asesorar y educar acerca del tema. A cada participante en el equipo se le proporcionó material necesario para que conociera y aprendiera acerca de los costos de calidad. Se le explicó los aspectos que rodean a los costos de calidad y la finalidad de los mismos. También se le entregó una copia del resumen de los elementos detallados del costo de Campanella (1992) para su familiarización con las categorías del modelo PEF y los elementos del costo.

Quinto paso, identificar las entradas y datos auxiliares al sistema. El equipo encontró los siguientes elementos como los generadores del costo en el área seleccionada. Estos elementos se pueden apreciar en el cuadro 2.

Sexto paso, desarrollar plan de ejecución. En base a este plan se obtendrán los costos de calidad e identificarán áreas de oportunidad dónde se podrán enfocar actividades impulsadas a mejorar y reducir los elementos generadores de costos encontrados. Se presenta este paso en la sección de resultados.

\section{RESULTADOS}

\subsection{Recolectar y cuantificar los costos}

El área piloto está formada por 10 células de producción y cada una está formada de ciertas estaciones en dónde se produce cantidades variadas de números de partes. Las células serán identificadas como se muestra en el cuadro 3.

Cadacélulafueabsorbiendoel costo correspondiente en cada elemento del costo identificado, con el fin de tener información necesaria para los análisis correspondientes.

Cuadro 2. Elementos de costo en el área piloto.

\begin{tabular}{|c|c|c|c|c|c|c|c|}
\hline Elemento & Prevención & Elemento & Evaluación & Elemento & Fallas Internas & Elemento & Fallas Externas \\
\hline $\mathrm{P} 1$ & Juntas diarias & E1 & $\begin{array}{l}\text { Revisión } \\
\text { inicial de } \\
\text { equipo }\end{array}$ & FI1 & Desperdicio & FE1 & $\begin{array}{l}\text { Quejas y } \\
\text { rechazos }\end{array}$ \\
\hline P2 & $\begin{array}{l}\text { Mantenimiento } \\
\text { preventivo del } \\
\text { equipo }\end{array}$ & E2 & $\begin{array}{l}\text { Auditorías de } \\
\text { calidad }\end{array}$ & $\mathrm{FI} 2$ & Retrabajo & FE2 & $\begin{array}{l}\text { Campañas con } \\
\text { el cliente }\end{array}$ \\
\hline P3 & $\begin{array}{l}\text { Capacitación para } \\
\text { la calidad }\end{array}$ & E3 & $\begin{array}{l}\text { Auditorías de } \\
\text { procesos }\end{array}$ & $\mathrm{FI3}$ & Tiempo Extra & & \\
\hline P4 & Auditorías 5's & E4 & $\begin{array}{l}\text { Pruebas } \\
\text { destructivas }\end{array}$ & $\mathrm{Fl} 4$ & Tiempo caído & & \\
\hline P5 & Auditorías TIR & E5 & $\begin{array}{l}\text { Pruebas de } \\
\text { resistencia }\end{array}$ & $\mathrm{FI5}$ & $\begin{array}{l}\text { Mantenimiento } \\
\text { correctivo }\end{array}$ & & \\
\hline P6 & Auditorías internas & E6 & $\begin{array}{l}\text { Pruebas de } \\
\text { penetración }\end{array}$ & FI6 & Inspección & & \\
\hline P7 & Mejora continua & E7 & Ultrasonido & $\mathrm{FI7}$ & Campañas & & \\
\hline P8 & $\begin{array}{l}\text { Carrera de la } \\
\text { calidad }\end{array}$ & E8 & $\begin{array}{l}\text { Análisis } \\
\text { dimensional }\end{array}$ & & & & \\
\hline
\end{tabular}

Fuente: Elaboración propia. 
Los costos se recolectaron en un lapso de tiempo de 5 semanas, tiempo suficiente para mostrar las variaciones en los costos en el área piloto. A continuación se explicará cómo se mostrarán los resultados de los costos obtenidos.

1. Se mostrará una tabla para cada categoría del costo de calidad.

2. Cada tabla mostrará los totales para las 5 semanas para cada elemento del costo.

3. Todos los costos están en pesos mexicanos.

Cuadro 3. Partes por célula de producción.

\begin{tabular}{|l|r|}
\hline \multicolumn{1}{|c|}{ Célula } & Cantidad de partes \\
\hline SIDE SILL & 4 \\
\hline COWL TOP F & 1 \\
\hline COWL TOP L & 1 \\
\hline TROUGH & 4 \\
\hline SHARK FIN & 6 \\
\hline FRONT BUMPER & 4 \\
\hline REAR BUMPER & 1 \\
\hline STRIKER & 8 \\
\hline PACKAGE TRAY & 2 \\
\hline LOWER BACK & 2 \\
\hline TOTAL & 33 \\
\hline
\end{tabular}

Fuente: Elaboración propia

En el cuadro 4 se muestran los costos relacionados a la prevención que fueron obtenidos en el área piloto durante el periodo de 5 semanas.

Cuadro 4. Costo total de prevención.

\begin{tabular}{|l|l|r|}
\hline Elemento & \multicolumn{1}{|c|}{ Concepto } & \multicolumn{2}{c|}{ Total } \\
\hline P1 & Juntas diarias & $\$ 14909$ \\
\hline P2 & Mantenimiento preventivo & $\$ 28316$ \\
\hline P3 & Capacitación en calidad & $\$ 88507$ \\
\hline P4 & Auditorías 5's & $\$ 1371$ \\
\hline P5 & Auditorías TIR & $\$ 444$ \\
\hline P6 & Auditorías internas & $\$ 1145$ \\
\hline P7 & Mejora continua & $\$ 1806$ \\
\hline P8 & Carrera de la calidad & $\$ 2442$ \\
\hline & Total de costos de prevención & $\$ 138941$ \\
\hline
\end{tabular}

Fuente: Elaboración propia.
En el cuadro 5 se muestran los costos relacionados a la evaluación que fueron obtenidos en el área piloto durante el periodo de 5 semanas.

Cuadro 5. Costo total de evaluación.

\begin{tabular}{|l|l|l|}
\hline Elemento & \multicolumn{1}{|c|}{ Concepto } & \multicolumn{1}{|c|}{ Total } \\
\hline E1 & Prueba de arranque diaria & $\$ 36650$ \\
\hline E2 & Auditoría de calidad & $\$ 57320$ \\
\hline E3 & Auditoría de procesos & $\$ 4705$ \\
\hline E4 & Pruebas destructivas & $\$ 70029$ \\
\hline E5 & Pruebas de resistencia & $\$ 39265$ \\
\hline E6 & Pruebas de penetración & $\$ 151231$ \\
\hline E7 & Ultrasonido & $\$ 36890$ \\
\hline E8 & Análisis dimensional & $\$ 187580$ \\
\hline & $\begin{array}{l}\text { Total de costos de } \\
\text { evaluación }\end{array}$ & $\$ 583670$ \\
\hline
\end{tabular}

Fuente: Elaboración propia.

En el cuadro 6 se muestran los costos relacionados a las fallas internas y que fueron obtenidos en el área piloto durante el período de 5 semanas.

Cuadro 6. Costo total de fallas internas.

\begin{tabular}{|c|c|c|}
\hline Elemento & Concepto & Total \\
\hline FI1 & Desperdicio & $\$ 42956$ \\
\hline $\mathrm{FI} 2$ & Retrabajo & $\$ 43189$ \\
\hline $\mathrm{FI3}$ & Tiempo extra & $\$ 84349$ \\
\hline FI4 & Tiempo caído & $\$ 226539$ \\
\hline $\mathrm{FI} 5$ & Mantenimiento correctivo & $\$ 183800$ \\
\hline FI6 & Inspección & $\$ 37650$ \\
\hline \multirow[t]{2}{*}{$\mathrm{FI} 7$} & Campañas & $\$ 4458$ \\
\hline & $\begin{array}{l}\text { Total de costos por fallas } \\
\text { internas }\end{array}$ & $\$ 622941$ \\
\hline
\end{tabular}

Fuente: Elaboración propia.

En el cuadro 7 se muestran los costos relacionados a las fallas externas y que fueron obtenidos en el área piloto durante el periodo de 5 semanas. 
Cuadro 7. Costo total de fallas externas.

\begin{tabular}{|l|l|c|}
\hline Elemento & \multicolumn{1}{|c|}{ Concepto } & Total \\
\hline FE1 & Quejas y rechazos & \$12 796 \\
\hline FE2 & Campañas con el cliente & \$3034 \\
\hline & $\begin{array}{l}\text { Total de costos por fallas } \\
\text { externas }\end{array}$ & \$15830 \\
\hline
\end{tabular}

Fuente: Elaboración propia.

\subsection{Analizar y detectar mejoras}

Una vez cuantificados y organizados los costos por categoría y elemento se procede a analizar la información en busca de áreas de oportunidad que promuevan la reducción de costos a través de la mejora continua.

Al registrar los costos en la categoría de prevención, evaluación y falla correspondiente se obtiene la siguiente información.

En el cuadro 8 se muestra el costo total de calidad desglosado por célula y por categoría, así mismo se muestra las ventas que servirán para el desarrollo del cuadro 9.

De esta forma se puede determinar que el costo total de calidad obtenido en el transcurso de 5 semanas es del $3.07 \%$ en base a las ventas totales, lo que equivale a $\$ 1361385.42$ pesos.

Cuadro 8. Costo total de calidad por célula y categoría.

\begin{tabular}{|c|c|c|c|c|c|c|}
\hline Célula & Prevención & Evaluación & $\begin{array}{c}\text { Fallas } \\
\text { Internas }\end{array}$ & $\begin{array}{c}\text { Fallas } \\
\text { Externas }\end{array}$ & $\begin{array}{l}\text { Total Costos } \\
\text { de Calidad }\end{array}$ & $\begin{array}{l}\text { Total de } \\
\text { Ventas }\end{array}$ \\
\hline SIDE SILL & $\$ 21595$ & $\$ 52801$ & $\$ 78098$ & $\$ 2274$ & $\$ 154768$ & \$14918441 \\
\hline COWL TOP F & 7598 & \$ 22185 & 853 & $\$ 1010$ & 31649 & \$ 1827496 \\
\hline COWL TOP L & $\$ \quad 8791$ & $\$ 22898$ & $\$ 9003$ & $\$ 1010$ & $\begin{array}{l}\quad 41702 \\
\end{array}$ & $\$ \quad 605237$ \\
\hline TROUGH & $\$ 15799$ & $\$ \quad 50927$ & $\$ 140277$ & $\$ \quad 1421$ & $\$ 208424$ & $\$ 5504901$ \\
\hline SHARK FIN & $\$ 8791$ & $\$ 63314$ & $\$ 47263$ & $\$ 1326$ & \$ 120694 & $\$ 5066219$ \\
\hline FRONT BUMPER & $\$ 34631$ & $\$ 173755$ & $\$ 173271$ & $\$ 4235$ & \$ 385892 & $\$ 7692579$ \\
\hline REAR BUMPER & \$ 14942 & $\$ 55779$ & \$ 68207 & $\$ 1010$ & \$ 139938 & \$ 3237317 \\
\hline STRIKER & $\$ 6656$ & $\$ 78155$ & \$ 28227 & $\$ 1257$ & \$ 114335 & $\$ 1722628$ \\
\hline PACKAGE TRAY & $\$ 10076$ & $\$ 31761$ & $\$ 33709$ & $\$ \quad 1188$ & 76734 & $\$ 1836494$ \\
\hline LOWER BACK & $\$ 10062$ & $\$ 32095$ & $\$ 43993$ & $\$ 1099$ & $\$ \quad 87249$ & $\$ 1957889$ \\
\hline Total Costos de Calidad & $\$ 138943$ & $\$ 583670$ & $\$ 622942$ & $\$ 15830$ & $\$ 1361385$ & \$ 44369201 \\
\hline
\end{tabular}

Fuente: Elaboración propia.

Cuadro 9. Costo total de calidad por categoría y célula en porcentaje.

\begin{tabular}{|l|r|r|r|r|r|}
\hline \multicolumn{1}{|c|}{ Célula } & \multicolumn{1}{|c|}{ Prevención } & \multicolumn{1}{c|}{ Evaluación } & Fallas Internas & \multicolumn{1}{c|}{$\begin{array}{c}\text { Fallas } \\
\text { Externas }\end{array}$} & $\begin{array}{c}\text { Total Costos } \\
\text { de Calidad }\end{array}$ \\
\hline SIDE SILL & $0.049 \%$ & $0.119 \%$ & $0.176 \%$ & $0.005 \%$ & $0.349 \%$ \\
\hline COWL TOP F & $0.017 \%$ & $v 0.050 \%$ & $0.002 \%$ & $0.002 \%$ & $0.071 \%$ \\
\hline COWL TOP L & $0.020 \%$ & $0.052 \%$ & $0.020 \%$ & $0.002 \%$ & $0.094 \%$ \\
\hline TROUGH & $0.036 \%$ & $0.115 \%$ & $0.316 \%$ & $0.003 \%$ & $0.470 \%$ \\
\hline SHARK FIN & $0.020 \%$ & $0.143 \%$ & $0.107 \%$ & $0.003 \%$ & $0.272 \%$ \\
\hline FRONT BUMPER & $0.078 \%$ & $0.392 \%$ & $0.391 \%$ & $0.010 \%$ & $0.870 \%$ \\
\hline REAR BUMPER & $0.034 \%$ & $0.126 \%$ & $0.154 \%$ & $0.002 \%$ & $0.315 \%$ \\
\hline STRIKER & $0.015 \%$ & $0.176 \%$ & $0.064 \%$ & $0.003 \%$ & $0.258 \%$ \\
\hline PACKAGE TRAY & $0.023 \%$ & $0.072 \%$ & $0.076 \%$ & $0.003 \%$ & $0.173 \%$ \\
\hline LOWER BACK & $0.023 \%$ & $0.072 \%$ & $0.099 \%$ & $0.002 \%$ & $0.197 \%$ \\
\hline Total Costos de Calidad & $0.31 \%$ & $1.32 \%$ & $1.40 \%$ & $0.04 \%$ & $3.07 \%$ \\
\hline
\end{tabular}

Fuente: Elaboración propia. 
Identificación de áreas de oportunidad para mejorar. En esta sección se muestra un caso de cómo detectar áreas de oportunidad y asignar prioridad a las acciones correctivas según el costo de los problemas. Se limitará solo a identificar y de ninguna manera se pretende dar solución a todos los problemas encontrados en el área piloto, debido a que la acción correctora se consigue mediante la utilización de otras herramientas.

De los datos de costos recolectados en el transcurso de 5 semanas, se puede determinar que las acciones correctivas enfocadas a los costos de fallas internas deberán establecerse como primera prioridad, seguido de los costos de evaluación. Los costos de prevención jugarán un papel muy importante ya que reforzarán y ayudarán a que se den y mantengan las acciones correctivas propuestas para mejorar los problemas encontrados en las fallas.

Porsu parte, los costos de fallas externas representan una cantidad mínima en relación a las otras categorías, por lo que pudiera considerarse como última prioridad pero sin mermar su importancia, dado que afecta directamente la satisfacción del cliente. De todas formas es de esperarse que este costo disminuya como consecuencia de mejorar las anteriores categorías del costo. A continuación se presentará un análisis detallado para costos de fallas internas.

Al hacer un análisis de fallas internas para determinar los contribuyentes de mayor costo, se encontraron los siguientes resultados que se muestran en el cuadro 10. Se puede determinar que los 3 principales contribuyentes de fallas internas son: tiempo caído, mantenimiento correctivo y tiempo extra. A continuación se realizará un análisis detallado de las causas que generan el tiempo caído.

En el cuadro 11 se muestran las causas principales que se encontraron en el área piloto. De las causas clasificadas se calculó que el $27 \%$ de ellas representan el $70 \%$ de los costos totales del tiempo caído. Para mejorar y reducir éstos costos se propone:

1. Como prioridad uno, se debe trabajar con el departamento de estampado para mantener el suministro de componentes para la operación de soldadura. Se deberán detectar las causas en esa área para bajar o eliminar esta problemática que cuesta en promedio de $\$ 6630$ pesos a la semana.

2. Preparar un plan de entrenamiento y capacitación para mejorar el tiempo de cambio de caps, boquillas, electrodos, pines y tambos de microalambre, debido a que se encontró variación significativa en cada evento de su tipo, lo que sugiere métodos diferentes de trabajo al realizar los cambios. Muy probablemente una formación para llegar a un método estándar podría disminuir los tiempos requeridos para estas operaciones y a su vez disminuir los problemas subsecuentes debido a un mal

Cuadro 10. Integración de los costos de fallas internas.

\begin{tabular}{|c|c|c|c|}
\hline Elemento & Fallas internas & $\%$ Ventas & Pareto de: \% Fallas internas / \%Total \\
\hline $\mathrm{Fl} 4$ & Tiempo caído & $0.511 \%$ & $36.37 \%$ \\
\hline FI5 & $\begin{array}{l}\text { Mantenimiento } \\
\text { correctivo }\end{array}$ & $0.414 \%$ & $29.51 \%$ \\
\hline $\mathrm{FI3}$ & Tiempo extra & $0.190 \%$ & $13.54 \%$ \\
\hline $\mathrm{Fl} 2$ & Retrabajo & $0.097 \%$ & $6.93 \%$ \\
\hline Fl1 & Desperdicio & $0.097 \%$ & $6.90 \%$ \\
\hline FI6 & Inspección & $0.085 \%$ & $6.04 \%$ \\
\hline \multirow[t]{2}{*}{$\mathrm{FI7}$} & Campañas & $0.010 \%$ & $0.72 \%$ \\
\hline & $\begin{array}{l}\text { Total costos de } \\
\text { fallas internas }\end{array}$ & $1.400 \%$ & \\
\hline
\end{tabular}

Fuente: Elaboración propia 
cambio. Esto ayudaría a reducir o eliminar un costo promedio de $\$ 11878$ pesos a la semana.

3. Encontrar la causa raíz y eliminar el problema de "falta de llave de reseteo". Esto costó \$4 258 pesos sólo porque alguien olvidó dejar la llave en su lugar.

4. Mejorar el plan actual de permisos de ir al baño a los operadores ya que cuesta alrededor de $\$ 900$ pesos a la semana, que de continuar así, representarían más de $\$ 40000$ pesos anuales.

5. Establecer un equipo de investigación para encontrar si hay una correlación entre las "fallas diversas" de los equipos, "ajustes e inspecciones varios", "teaching" y pruebas de "pokayoke", ya que a través de las costos se puede interpretar que quizás manos inexpertas pudieran estar moviendo o estableciendo parámetros incorrectos que conlleven a la gran cantidad de minutos en tiempo caído por estas categorías. Un buen plan de entrenamiento con los proveedores de los equipos pudiera disminuir los $\$ 7288$ pesos que se gastan a la semana por estos conceptos.
6. Analizar las causas de los costos relacionados por culpa del proveedor y corroborar con el área de finanzas para que correctamente le facture a los proveedores los gastos relacionados al tiempo caído y que son atribuidos a ellos. Los costos ascienden a \$3 167 pesos en total.

7. Establecer un método estándar y definir códigos únicos para cuando se reporte el tiempo caído, esto debido a que actualmente una misma causa de tiempo caído se clasifica con diferentes códigos lo que dificulta y hace más tardada la tarea de detección de los problemas de esta índole.

\section{CONCLUSIONES}

Al obtener los costos de calidad utilizando el modelo PEF en el área de piloto de la empresa se pudo cumplir con los objetivos específicos planteados, ya que se:

- Identificaron y clasificaron cada uno de los elementos del costo de calidad existentes en el área de producción de soldadura.

Cuadro 11. Causas de tiempo caído.

\begin{tabular}{|c|c|c|c|}
\hline Causas del tiempo caído & Minutos & Costo Total & $\%$ de Ventas \\
\hline Falta material del área de estampado & 5840 & $\$ 33147.03$ & $0.075 \%$ \\
\hline Cambio / problemas con caps & 2645 & $\$ 20263.98$ & $0.046 \%$ \\
\hline Pruebas pokayoke & 1998 & $\$ 14973.19$ & $0.034 \%$ \\
\hline Cambio / problemas con electrodos & 2170 & $\$ 14366.76$ & $0.032 \%$ \\
\hline Cambio / problemas con boquillas & 2007 & $\$ 11038.38$ & $0.025 \%$ \\
\hline Fallas diversas & 1337 & 9964.71 & $0.022 \%$ \\
\hline Cambio / problemas con pines & 1866 & 8403.68 & $0.019 \%$ \\
\hline Limpieza por rebabas & 889 & 6124.78 & $0.014 \%$ \\
\hline Ajustes e inspecciones varios & 850 & 5787.71 & $0.013 \%$ \\
\hline Teaching & 777 & 5718.92 & $0.013 \%$ \\
\hline Problemas con cordones & 808 & 4537.09 & $0.010 \%$ \\
\hline Operador al baño & 795 & 4504.48 & $0.010 \%$ \\
\hline Falta llave reseteo & 467 & 4258.31 & $0.010 \%$ \\
\hline Cambio / problemas con microalambre & 622 & 3582.67 & $0.008 \%$ \\
\hline Culpa del proveedor & 440 & 3167.88 & $0.007 \%$ \\
\hline Retrabajos en celda & 370 & 2844.37 & $0.006 \%$ \\
\hline Problemas con componentes o surtido & 442 & 2772.25 & $0.006 \%$ \\
\hline Puntos fríos / pequeños & 214 & 2248.52 & $0.005 \%$ \\
\hline Fugas de agua & 162 & 1737.16 & $0.004 \%$ \\
\hline Otros problemas & 9731 & $\$ 67097.31$ & $0.151 \%$ \\
\hline TOTAL & 34430 & $\$ 226,539.18$ & $0.511 \%$ \\
\hline
\end{tabular}

Fuente: Elaboración propia 
- Costearon o estimaron los elementos del costo de calidad identificados mediante una base y metodología contable que puede utilizarse en la empresa.

- Seleccionaron bases o índices financieros relacionados al costo de calidad que permitirán la toma de decisiones justificada para la mejora y comparación con otras empresas.

Asimismo, se pudo lograr el objetivo general al:

- Desarrollar un sistema de costos de calidad piloto utilizando el modelo PEF enfocado al área de producción de soldadura de la empresa.

- El modelo PEF es aplicable al área de soldadura y otorgará una visión de los problemas en términos monetarios como un común denominador que permitirá a la gerencia de la empresa designar prioridad a los proyectos de mejora que se lleven cabo a través de un análisis y justificación monetaria.

La implementación del modelo PEF en el área de soldadura traerá consigo un beneficio cualitativo en la toma de decisiones, sólo a través de cuándo, quién y cómo se interpreten los datos y cómo se tomen y ejecuten las decisiones se verán reflejados los beneficios de ahorros que se obtendrán con la mejora continua.
Este modelo de costos de calidad es sin duda una excelente herramienta administrativa que permite encontrar áreas de oportunidad traduciendo los problemas a dinero, unificando las diferentes variables objetivas y subjetivas en un mismo término cuantificable y entendible.

\section{REFERENCIAS}

[1] Atkinson, J.H. (1991). Current trends in cost of quality: linking the cost of quality and continuous improvement. Montvale, New Jersey: National Association of Accountants .

[2] Campanella, J. (1992). Principios de los costes de la calidad. Madrid: Ediciones Díaz de Santos.

[3] Dale, B.G. y Plunkett, J.J. (1991). Costos en la calidad. Grupo Editorial Iberoamérica.

[4] Harrington, H.J. (1999). Performance improvement: a total poor-quality cost system. The TQM Magazine, 11(4), pp.221-230.

[5] Juran, J.M. y Gryna, F.M. (1993). Quality planning and analysis: from product development through use. McGraw-Hill. 